\title{
Essential medicines for children in Armenia
}

\author{
I. Kazaryan ${ }^{\mathrm{a}}$ and L. Vardanyan ${ }^{\mathrm{b}, *}$ \\ a Drug Utilization Research Group PO, Yerevan, Armenia \\ ${ }^{\mathrm{b}}$ Pharmaceutical Management, Yerevan State Medical University, Yerevan, Armenia
}

*Corresponding author. E-mail: vlus69@mail.ru

BACKGROUND: Drug therapy plays a key role in pediatrics. Effective treatment in pediatric practice means that medicines should be both effective and safe for children. However, there is not enough data on effectiveness and safety of medicines for children due to small number of clinical trials [1]. Access to medicines for children remains a serious problem worldwide [2]. The World Health Organization (WHO) has significantly increased the efforts in the field of pediatric pharmaceuticals in order to improve the situation [4]. In 2007, the World Health Organization (WHO) Model List of Essential Medicines for Children (EMLc) was firstly developed. Although WHO promotes access to essential medicines for children in countries by encouraging inclusion of these medicines in national essential medicines lists (EMLs) and treatment guidelines, many essential medicines are not covered by (EMLs) in some countries. The results of a study performed in 14 countries has shown that of 20 surveyed medicines the proportion included in national EMLs ranged from 50\% to $90 \%$ and some medicines of the EML were not included in local standard treatment guidelines [3].

OBJECTIVES: To examine the current situation with access to essential medicines for children in the Republic of Armenia (RA).

METHODS: The Armenian Essential Medicines Lists (AEML) of 2007 and 2013, as well as the Lists of medicines registered in Armenia have been analyzed (2011 - 2013). The following indicators have been calculated: the percentage of medicines from the WHO EMLc, which were included on the current AEML, the percentage of medicines from the WHO EMLc which were registered in Armenia and the percentage of medicines from the WHO EMLc which were included in approved clinical guidelines. Also, recommendations on prescribing of medicines for pneumonia, included in Armenian clinical guidelines, were analyzed.

RESULTS: The analysis of the use of pharmaceuticals from the WHO Model List of Essential Medicine for Children (WHO EMLc) by medicines supply system in the Armenia has revealed that in 2013 only $57.7 \%$ of all the medicines from WHO EMLc were included on the National List of Essential Medicines of RA (AEML) and only 68.5\% were registered. The results of studies carried out in 2011, 2012, 2013 showed that the situation in regard to the coverage of medicines from the WHO EMLc (without taking into account drug formulations and strengths) by AEML and clinical guidelines used in RA has not changed considerably during that period of time, while the percentage of essential medicines for children recommended by WHO, which were registered in Armenia and available in the pharmaceutical market, slightly increased. 
Analysis of recommendations on prescribing medicines for pneumonia treatment, included in clinical guidelines used in Armenia, has revealed that these recommendations slightly differ in terms of recommended medicines and other treatment details. Accordingly, approval and use of a single set of criteria for selecting medicines to be included into pediatric clinical guidelines should be considered as an effective approach for optimizing supply of medicines for children.

CONCLUSIONS: Access to essential medicines for children in Armenia is unsatisfactory. Essential medicines for children recommended by the WHO are only partially covered by the medicines supply system for children in Armenia. Development of the List of Essential Medicines for children in Armenia is an approach to solve this problem. Currently actions are being taken in this direction. It is also considered appropriate to develop national clinical guidelines on the most common childhood diseases in Armenia.

Keywords: Access to medicines, essential medicines, children, clinical guidelines, Armenia

\section{References}

[1] Finney E. Children's medicines: A situational analysis. World Health Organization, Geneva, 2011.

[2] Ivanovska V., Mantel-Teeuwisse Aukje K., Dijk L.Van. Background Paper 7.1 Priority Medicines for Children. WHO Collaborating Centre for Pharmaceutical Policy and Regulation, Utrecht, the Nitherlands, 2013.

[3] Robertson J., Forte G., Trapsida J.-M., Hill S. What essential medicines for children are on the shelf? Bull World Health Organization. 2009;87:231-37.

[4] WHO Better medicines for children. Sixtieth World Health Assembly, Geneva, 2007. 